\title{
Off-Shell Supersymmetry versus Hermiticity in Superstrings
}

\author{
Nathan Berkovits* \\ Instituto de Física Teórica, Universidade Estadual Paulista, Rua Pamplona 145, São Paulo, SP 01405-900, Brazil
}

(Received 3 June 1996)

\begin{abstract}
We point out that off-shell four-dimensional spacetime supersymmetry implies strange Hermiticity properties for the $N=1$ Ramond-Neveu-Schwarz superstring. However, these Hermiticity properties become natural when the $N=1$ superstring is embedded into an $N=2$ superstring. [S00319007(96)01339-7]
\end{abstract}

PACS numbers: $11.25 . \mathrm{Db}$

Superstring theory is the only known renormalizable quantum theory of gravity, and spacetime supersymmetry plays a crucial role in removing the divergences. Spacetime supersymmetry also plays a crucial role in the recent conjectures [1] of strong-weak duality in superstring theory. However, spacetime supersymmetry is only an on-shell symmetry in the conventional $N=1$ RamondNeveu-Schwarz (RNS) description of the superstring, which makes it difficult to observe its effects.

It will be shown in this Letter that after modifying the conventional Hermiticity properties, four-dimensional supersymmetry can be extended to an off-shell symmetry of the superstring. The modified Hermiticity properties are those of an $N=2$ embedding of the $N=1$ RNS superstring [2], where $N=1$ and $N=2$ refer to the number of worldsheet superconformal invariances. This suggests that the $N=2$ description of the superstring [3] may be useful for illuminating the effects of spacetime supersymmetry. It is interesting to note that $N=2$ superstrings have recently appeared in models [4] which try to explain the strong-weak duality symmetries.

In four-dimensional compactifications of the $N=1$ RNS superstring, the spacetime-supersymmetry generators in the $-\frac{1}{2}$ picture are

$$
q_{a}=\frac{1}{2 \pi i} \oint d z e^{(1 / 2)\left(-\phi \pm i \sigma_{0} \pm i \sigma_{1} \pm i H_{C}\right)},
$$

where there are an even number of + signs in the exponential $(a=1$ to 4$), \phi$ comes from fermionizing the bosonic ghosts as $\beta=i \partial \xi e^{-\phi}$ and $\gamma=-i \eta e^{\phi}, \psi^{3}=$ $\pm \psi^{0}=e^{ \pm i \sigma_{0}}$ and $\psi^{1} \pm i \psi^{2}=e^{ \pm i \sigma_{1}}$ where $\psi^{m}$ is the fermionic vector, and $\partial H_{C}=J_{C}$ is the $\mathrm{U}(1)$ generator of the $c=9, N=2$ superconformal field theory representing the compactification manifold.

These spacetime-supersymmetry generators satisfy the anticommutation relations

$$
\left\{q_{a}, q_{b}\right\}=\frac{1}{2 \pi i} \oint d z e^{-\phi} \psi_{m} \gamma_{a b}^{m}
$$

which is not the usual supersymmetry algebra $\left\{q_{a}, q_{b}\right\}=$ $\frac{1}{2 \pi} \oint d z \partial x_{m} \gamma_{a b}^{m}$ where $\frac{1}{2 \pi} \oint d z \partial x_{m}$ is the string momentum. However, after hitting the right-hand side of (2) with the picture-changing operator $Z=\{Q, \xi\}$, it becomes $\frac{1}{2 \pi i} \oint d z Z e^{-\phi} \psi_{m} \gamma_{a b}^{m}=\frac{1}{2 \pi} \oint d z \partial x_{m} \gamma_{a b}^{m}$. So up to picture changing, the $q_{a}$ 's form a supersymmetry algebra [5].

But off-shell supersymmetry requires that the $q_{a}$ 's form a supersymmetry algebra without applying picturechanging operations. This is because picture changing is only well defined when the states are on-shell. Offshell, the states are not independent of the locations of the picture-changing operators.

So off-shell spacetime supersymmetry requires modification of the $q_{a}$ 's. Note that $q_{a}$ has picture $-\frac{1}{2}$ and the momentum $\frac{1}{2 \pi} \oint d z \partial x_{m}$ has picture 0 , so we need generators with picture $+\frac{1}{2}$. The obvious solution [3] is to split $q_{a}$ into a chiral part with picture $-\frac{1}{2}$ and an antichiral part with picture $+\frac{1}{2}$ :

$$
\begin{aligned}
q_{\alpha}= & \frac{1}{2 \pi i} \oint d z e^{(1 / 2)\left[-\phi \pm i\left(\sigma_{0}+\sigma_{1}\right)+i H_{C}\right]}, \\
\bar{q}_{\dot{\alpha}}= & Z q_{\dot{\alpha}}=\frac{1}{2 \pi i} \oint d z\left[b \eta e^{(1 / 2)\left[3 \phi \pm i\left(\sigma_{0}-\sigma_{1}\right)-i H_{C}\right]}\right. \\
& +i:\left(e^{\phi} \psi_{m} \partial x^{m}+e^{\phi} G_{C}^{+}+e^{\phi} G_{C}^{-}\right) \\
& \left.\times e^{(1 / 2)\left(-\phi \pm i\left(\sigma_{0}-\sigma_{1}\right)-i H_{C}\right.}:\right],
\end{aligned}
$$

where $G_{C}^{ \pm}$are the fermionic generators of the $c=9$ $N=2$ superconformal field theory. The $N=14 \mathrm{D}$ supersymmetry algebra $\left\{q_{\alpha}, \bar{q}_{\dot{\beta}}\right\}=\frac{1}{2 \pi} \oint d z \partial x_{m} \sigma_{\alpha \dot{\beta}}^{m}$ is now satisfied off-shell where we are using standard two-component Weyl notation.

Although we have solved the problem of finding offshell supersymmetry generators, we now have a new problem. Using the standard RNS definition of Hermiticity where all fundamental fields are Hermitian or antiHermitian (the anti-Hermitian field is $\sigma_{0}$ ), the Hermitian conjugate of $q_{\alpha}$ is no longer $\bar{q}_{\dot{\alpha}}$. Fortunately, this new problem can be solved by modifying the definition of Hermiticity. However, this new Hermiticity definition will be natural only if one embeds the $N=1$ superstring into an $N=2$ superstring.

To find the appropriate Hermiticity definition, one first writes $\bar{q}_{\dot{\alpha}}$ in the form

$$
\bar{q}_{\dot{\alpha}}=e^{R}\left(\frac{1}{2 \pi i} \oint d z b \eta e^{(1 / 2)\left[3 \phi \pm i\left(\sigma_{0}-\sigma_{1}\right)-i H_{C}\right]}\right) e^{-R},
$$

1996 The American Physical Society 
where

$$
R=\frac{1}{2 \pi} \oint d z c \xi e^{-\phi}\left(\psi^{m} \partial x_{m}+e^{\phi} G_{C}^{+}+G_{C}^{-}\right)
$$

and $e^{R} F e^{-R}=F+[R, F]+\frac{1}{2}[R,[R, F]]+\cdots$ (the expansion usually stops after two terms).

One then defines Hermiticity as

$$
\begin{array}{rlrl}
\left(x_{m}\right)^{\dagger} & =e^{R} x_{m} e^{-R}, \quad\left(\psi_{m}\right)^{\dagger}=e^{R} \psi_{m} e^{-R}, \quad\left(F_{C}\right)^{\dagger}=e^{R} \bar{F}_{C} e^{-R}, \\
\left(e^{\phi / 2}\right)^{\dagger} & =e^{R}\left(c \xi e^{-(3 / 2) \phi}\right) e^{-R}, & & \left(e^{-\phi / 2}\right)^{\dagger}=e^{R}\left(b \eta e^{(3 / 2) \phi}\right) e^{-R}, \\
(b)^{\dagger} & =e^{R}\left(i b \eta \partial \eta e^{2 \phi}\right) e^{-R}, & & (c)^{\dagger}=e^{R}\left(-i c \xi \partial \xi e^{-2 \phi}\right) e^{-R}, \\
(\eta)^{\dagger} & =e^{R}\left(i \eta b \partial b e^{2 \phi}\right) e^{-R}, & & (\xi)^{\dagger}=e^{R}\left(-i \xi c \partial c e^{-2 \phi}\right) e^{-R},
\end{array}
$$

where $F_{C}$ are the worldsheet fields in the $c=9, N=2$ superconformal field theory. It is straightforward to check that the new Hermiticity definition satisfies $\left(F^{\dagger}\right)^{\dagger}=F$ for all $F$, preserves OPE's, and implies that $\left(q_{\alpha}\right)^{\dagger}=\bar{q}_{\dot{\alpha}}$.

One strange feature of the Hermiticity definition of (5) is that a field may have a different conformal weight from its Hermitian conjugate since $(T)^{\dagger}=T+i \partial(b c+\xi \eta)$ where $T$ is the RNS Virasoro generator. Another strange feature is that the BRST operator is not Hermitian since $Q^{\dagger}=\frac{1}{2 \pi} \oint d z b$. [This easily follows from writing $Q=$ $e^{R}\left(\frac{i}{2 \pi} \oint d z b \eta \partial \eta e^{2 \phi}\right) e^{-R}$.]

Although these features are strange in the $N=1$ RNS description of the superstring, they are natural if the $N=$ 1 superstring is embedded into an $N=2$ superstring. As discussed in Ref. [2], any critical $N=1$ superstring can be embedded into a critical $N=2$ superstring where the $c=6, N=2$ superconformal generators are [2]

$$
\begin{gathered}
T_{N=2}=T_{N=1}+\frac{i}{2} \partial(b c+\xi \eta), \\
G_{N=2}^{+}=j_{\text {BRST }}, \quad G_{N=2}^{-}=b, \quad J_{N=2}=b c+\xi \eta,
\end{gathered}
$$

and $j_{\mathrm{BRST}}=e^{R}\left(i b \eta \partial \eta e^{2 \phi}\right) e^{-R}$.
Using the Hermiticity definition of (5), $\left(T_{N=2}\right)^{\dagger}=$ $T_{N=2},\left(G_{N=2}^{+}\right)^{\dagger}=G_{N=2}^{-}$, and $\left(J_{N=2}\right)^{\dagger}=J_{N=2}$, which are the standard Hermiticity properties of an $N=2$ string. [This Hermiticity can be made manifest by writing the $N=2$ generators of (6) in terms of spacetimesupersymmetric variables [3].] So the Hermiticity properties implied by off-shell four-dimensional supersymmetry are natural only if the $N=1$ superstring is embedded into an $N=2$ superstring.

*Electronic address: nberkovi@snfma2.if.usp.br

[1] A. Sen, Int. J. Mod. Phys. A 9, 3707 (1994); P. K. Townsend, Phys. Lett. B 350, 184 (1995); E. Witten, Nucl. Phys. B443, 85 (1995); C. M. Hull, Nucl. Phys. B468, 113 (1996).

[2] N. Berkovits and C. Vafa, Mod. Phys. Lett. A 9, 653 (1994).

[3] N. Berkovits, Nucl. Phys. B431, 258 (1994).

[4] M. B. Green, Nucl. Phys. B293, 593 (1987); C. Vafa, Report No. hep-th/9602022; D. Kutasov and E. Martinec, Report No. hep-th/9602049.

[5] D. Friedan, E. Martinec, and S. Shenker, Nucl. Phys. B271, 93 (1986). 